\title{
DISCUTIENDO ALGUNAS ESCALAS DE LA TRANSMISIÓN CULTURAL: ARTEFACTO Y ESPACIO
}

\author{
José Luis Lanata* \\ Hector Neff**
}

Durante los últimos 20 años, los enfoques evolutivos han tomado un lugar destacado en la Arqueología moderna (Dunnell 1978a, 1980, 1989, 1992a y b, 1994; Kirch 1980; Rindos 1984, 1985, 1989a y b; Leonard y Jones 1987; Foley 1989; Smith 1991; O’Brien \& Holland 1990 y 1992; Smith \& Winterhalder 1992; Neff 1992; Lanata 1993a; Teltser (ed.) 1995; O’Brien 1996; Maschner (ed.) 1996, Barton y Clark (eds.) 1997; Lyman y O’Brien 1998 , tan solo por citar algunos trabajos). Sin duda analizar el cambio del género Homo implica atacarlo en sus dos expresiones, la genética y la cultural.

En este trabajo discutimos la transmisión de rasgos y como el artefacto y el espacio son vistos en la Arqueología Evolutiva. Específicamente queremos comenzar la discusión de estas escalas de análisis - artefacto y espacio - en la transmisión en nuestro campo.

\section{Tema central: el cambio}

La evolución de las poblaciones humanas ha sido desde sus inicios uno de los temas principales que ha abordado la Arqueología, y en especial a partir del inicio del siglo XX. Sin embargo, no es hasta hace unos pocos años en que comenzamos a sentir la necesidad de estudiar el cambio más allá de las corrientes teórico-metodológicas dominantes en Arqueología desde inicios de la década de 1960. Si bien la New Archaeology introdujo y extendió el uso de los conceptos de adaptación y proce-

(*) Departamento de Antropología, Universidad de Buenos Aires y PREP-CONICET. Buenos Aires - Argentina. (**) $230 Z$ Research Reactor Center, University of Missouri-Columbia, USA. sos adaptativos, esto no significó un cambio paradigmático en cuanto al ataque del tema (Dunnell 1980). Desde el comienzo de la década del ' 80 los trabajos orientados teóricamente a la aplicación de la Teoría de la Evolución al registro arqueológico han ocupado un lugar importante en los medios académicos. ${ }^{1}$ Uno de los puntos más destacables - y en donde el acuerdo entre los científicos es unánime - es que la aplicación de la Teoría de la Evolución en Arqueología no puede reducirse a una simple analogización de sus principios en nuestro campo. Muy por el contrario, la necesidad de explicar la variación del registro arqueológico a través de la aplicación de la Teoría de la Evolución requiere de adecuados métodos analíticos (Leonard y Jones 1987) y comparativos (Neff y Larson 1997). Esta nueva corriente teórico-metodológica - con algunas diferencias entre sus seguidores - es lo que en grandes rasgos conocemos como Arqueología Evolutiva (ver las distintas corrientes en Lanata 1998 y Lyman y O'Brien 1998).

La Arqueología Evolutiva ha colocado especial énfasis en los distintos aspectos de la selección (Dunnell 1989; Rindos 1989; O'Brien y Holland 1990 y 1995a; Jones et al. 1995). Rindos (1989) y Dunnell (1989) advirtieron que la generación de variación es un paso previo y separado al de la selección de un determinado rasgo. Dunnell (1980) señala que la evolución posee tres componentes críticos: a) variabilidad empírica, b) persistencia o transmisión de parte o de toda la variabilidad y

(1) El lector puede recurrir a Teltser ed. 1995, O'Brien 1996, Maschner ed. 1996, Barton y Clark ed. 1997 para obtener un ejemplo de los trabajos generados dentro de este cuerpo teórico en Arqueología. Recientes discusiones y relaciones entre diferenes enfoques teóricos pueden verse en Schiffer 1996, Spencer 1997, Boone y Smith 1998 y Lyman y O'Brien 1998. 
c) representación diferencial de la variabilidad transmitida en los estados siguientes. Como resultado de la variabilidad se produce una persistencia diferencial específica e intra-específica, tanto en genotipos como en fenotipos - con tasas de reproducción diferenciales - en las sucesivas generaciones. A lo largo del tiempo, esta diversidad está representada por la presencia de diferentes taxas. La relación evolutiva entre ambiente y taxon produce distribuciones particulares y en continuo cambio en espacio y tiempo de estos organismos. Esto es lo que llamamos selección darwiniana.

A diferencia de lo mencionado anteriormente, la visión lamarckiana implica creer en una intencionalidad en el proceso de selección. Esta visión - más antropológica - normalmente confunde intención con selección, reforzando la idea de finalidad en el proceso evolutivo. Esta diferenciación entre selección lamarckiana y darwiniana sin duda nos coloca en un dilema. ¿Es la cultura un fenómeno aparte dentro del reino animal, y por lo tanto opera diferente que la evolución biológica? Dawkins (1982) presenta una interesante idea sobre las propiedades de los fenotipos. Como continuación del concepto de 'selfish gene' (Dawkins 1976), postula que cada gen no es seleccionado directamente sino, en parte, por su efecto fenotípico. Así, al producirse la transmisión de los caracteres, puede pensarse que también tiene "extended phenotypic effects, consisting of all its effects on the world at large, not just its effects on the individual body in which it happens to be sitting" (Dawkins 1982:4, énfasis en el original). Boyd y Richerson (1985) postularon que los fenotipos pueden verse como la interacción entre la información transmitida y el ambiente. Así, los artefactos - en un sentido amplio - representan medios a través de los cuales los homínidos se 'relacionan' con el medio, en otras palabras, son el producto de la interacción entre la información genética y/o cultural y el ambiente (Smith 1991). Dunnell (1989:44) expresa que los "artifacts are the hard parts of the behavioral segment of phenotypes". en los seres humanos. Esta idea ha sido desarrollada por algunos arqueólogos en los últimos años (Dunnell 1989, 1992a; Foley 1989; Rindos 1989; Smith 1991; O'Brien y Holland 1990, 1992, 1995b; O'Brien et al. 1994), concibiendo a los artefactos como parte del fenotipo de las distintas especies de Homínidos.

\section{Selección y transmisión}

En el proceso de generar variación y seleccionar determinados rasgos nos enfrentamos, como arqueólogos, a la necesidad de reconocer los diferentes niveles en los que se da; el genético y el cultural. Y esto básicamente nos remite a ver que ambos niveles son diferentes y operan bajo tempos y modos particulares. Si como hemos mencionado antes, los arqueólogos evolutivos hemos prestado atención desde hace casi dos décadas atrás a cómo opera la selección - ver supra - no es hasta hace poco tiempo que comenzamos a trabajar sobre la transmisión de rasgos (Bettinger 1991, Bettinger y Eerkens 1997, Shott 1997, Neff 1998, aunque Dunnell 1978a es una excepción). ${ }^{2}$ Años atrás, Dunnell (1978b [1996]) propuso que la transmisión cultural da una oportunidad al nivel de selección de cambiar desde el nivel del individuo hacia uno más amplio, aquél que en forma colectiva integra funcionalmente a los individuos. En varias oportunidades Dunnell (i.e. 1989) ha mencionado esta posibilidad especialmente en el caso del comienzo de las sociedades complejas. El caso opuesto - que la selección actúe a un nivel por debajo del individuo - no ha sido discutido en la Arqueología Evolutiva sin embargo ver Neff 1998. Esta omisión es muy sorprendente considerando que:

a) ha habido una considerable discusión en cuanto a cómo los artefactos pueden exhibir un fitness diferencial (i.e. Neff y Larson 1997; O'Brien y Holland 1990, 1992)

b) que los artefactos pueden exhibir descendencia con modificaciones a pesar de que los individuos que los usan se reproduzcan exitosamente o no, $y$

c) que los esfuerzos prácticos en el uso de la Teoría de la Evolución puede tener sentido en el registro arqueológico, invariablemente se foca-

(2) La generación y/o uso de artefactos no es dominio de una sola especie. Así como biólogos, etólogos y taxónomos estudian distintas características fenotípicas de las especies, también presentan debida atención a los artefactos que éstas fabrican y/o utilizan (Bonner 1980, Griffin 1984, Walker 1983, McGrew 1992 y 1993, Visalberghi 1993). No discutiremos aquí las diferencias en los modos de transmisión entre Homo (i.e. Cavalli-Sforza y Feldman 1981, Boyd y Richerson 1985) y otros géneros relacionados (Boesch 1993). 
LANATA, J.L.; NEFF, H. Discutiendo algunas escalas de la transmisión cultural: artefacto y espacio. Rev. do Museu de Arqueologia e Etnologia, São Paulo, Suplemento 3: 47-56, 1999.

lizan en el artefactos (i.e. Dunnell y Feathers 1991, Neff y Arroyo n.d., O'Brien et al. 1994).

Dunnell (1978a y b) remarca que el gran aumento en la tasa de cambio y en la escala de la diversidad es posible tras la invención de la transmisión cultural. Dunnell equipara esto con la invención de la reproducción sexual y la consecuente "Explosión Cámbrica" de la diversidad biológica. De acuerdo con Dunnell (1978a: 198) el impacto del sexo y la transmisión cultural son comparables ya que ambas acortan el tiempo de las respuestas adaptativas e incrementan su rango - es decir su variabilidad. Pero, ya que la respuesta adaptativa es enteramente el resultado de la selección (O'Brien y Holland 1990, Neff y Larson 1997), la observación de Dunnell nos lleva a preguntarnos por que la invención del sexo y la transmisión cultural proveen a la selección una oportunidad para actuar más rápidamente y ser más flexible.

Neff (1998) ha sugerido que la respuesta es que ambas invenciones - la reproducción sexual y la transmisión cultural - conllevan a un cambio en la relación entre los replicadores por un lado, y los que Hull (1980) llama interactors y Dawkins (1976 y 1982) vehicles, por el otro. En todos los organismos anteriores a la Explosión Cámbrica, el genoma entero de los mismos es un replicator, y el organismo en el cual reside es el vehicle. El organismo-linaje es un constante árbol divergente que paraleliza precisamente el genoma-linaje. La selección se da entre organismo-linajes y produce frecuentes cambios en la representación del genoma-linaje. Si una nueva característica que aparece por mutación incrementa la habilidad de un organismo para asegurarse los recursos necesarios, los genomas determinados por esas características incrementarán en frecuencia tanto como el organismo-linaje desarrolla linajes derivados más rápidamente.

El panorama cambió con la invención del sexo. Los organismos individuales se convierten en vehículos para múltiples replicadores, y estos replicadores pueden ahora ser identificados como genes individuales más que como genomas completos. Los replicadores se asocian con otro, ambos temporalmente, en vehículos individuales, y más permanentemente en el pool genético de las especies. Es este, el pool genético de la especie, el que muestra continuidad a lo largo del tiempo y que define el linaje evolutivo, pero este no es el nivel en el que actúa la selección. La selección actúa en el nivel del interactor - o del vehículo. Lo que sucede es que los genes de la especielinaje son recambiados en cada generación en un único, nuevo set de interactores. La selección tiene lugar porque el fitness diferencial entre expresiones fenotípicas alternativas que han emergido en un tiempo particular a través de la división y clasificación de genes basada en los sexos y de los procesos de desarrollo resultantes desde el estado zigota hasta el adulto.

Desde esta perspectiva, la invención del sexo cambia por definición el nivel de selección hacia uno más abajo. Más que tomar lugar en el nivel de linaje - como lo hace en los organismos asexuados - la selección en los organismos con reproducción sexual esta basada en el fitnesses discordante de expresiones fenotípicas alternativas dentro del linaje. En el segundo caso, la selección actúa mucho más rápido y en forma más flexible, y es esto lo que cuenta en la explosión de la diversidad biológica durante el período Cámbrico que Dunnell (1978a) menciona.

Ahora, ¿cómo es que la emergencia de la transmisión cultural afecta el proceso de selección y, específicamente, el nivel en el cual este tiene sus efectos primarios? Tal como la emergencia de la reproducción sexual, la naturaleza tanto de los replicadores como de interactores cambia. Los replicadores consisten en información cultural que viene en forma de paquete - i.e. como memes, culturgens, etc. - y transmitidas de un individuo a otro. Es posible pensar también que esos paquetes de información como pools culturales que muestran cierta continuidad a lo largo de un tiempo y de un espacio dados y define el linaje cultural evolutivo. Un punto clave en esto es que la transmisión cultural es independiente de la genética (Neff 1998). Los linajes evolutivos culturales son completamente independientes de los linajes genéticos. Pero ¿qué define el interactor, la manifestación fenotípica de la información del pool cultural? Esta es la pregunta clave, porque es el éxito diferencial de los interactores lo que le da la oportunidad a la selección para dar forma al pool de variación cultural.

Dentro de la Arqueología Evolutiva, la tendencia ha sido pensar en el individuo y el grupo de individuos como los únicos dos candidatos posibles para actuar como interactores (i.e. Dunnell 1978a), lo que nos parece extraño. Tanto el individuo como el grupo pueden adoptar alternativas, 
mutuamente exclusivas de rasgos culturales. Por ejemplo, cuando mucho de nosotros comenzamos nuestras carreras en Arqueología, no había computadoras personales, mientras que ahora ninguno podría pensar en trabajar sin ellas. ¿Cuál de estas alternativas - el uso de computadoras personales y el no-uso - deben ser consideradas para caracterizar el fenotipo de un arqueólogo particular o algún grupo de arqueólogos? Dado que este rasgo - la computadora personal - es culturalmente heredado puede existir serialmente en estados alternativos dentro del mismo individuo o del grupo, es claro que ni el individuo ni el grupo pueden ser considerados como interactors en este caso. Por lo tanto este rol cae en el rasgo en si mismo. El rasgo es la manifestación fenotípica de un set coherente de instrucciones culturales, y su éxito relativo o su fracaso determinan tanto el incremento como el descenso de las instrucciones culturales en frecuencia en el pool cultural. Este proceso no tiene nada que ver con el éxito y el ciclo reproductivo de los individuos. El no uso total de las computadoras personales desaparece del pool cultural en casi el período de vida de un individuo, y esto no tiene completamente nada que ver con el éxito reproductivo diferencial (Neff 1998).

Obviamente, el pool genético humano y el pool cultural son diferentes. Neff (1998) ha señalado que es totalmente erróneo haber mantenido el mismo interactor - el individuo humano - en la persistencia diferencial de la variación de ambos pools. Por un lado, podemos estar bastante seguros que el éxito reproductivo diferencial de los individuos humanos puede conducir a cambios en la frecuencia genética. Pero atar la posibilidad de cambio en la frecuencia de las instrucciones culturales al éxito reproductivo diferencial es absurdo, como el ejemplo de las computadoras personales lo mostró.

Este tema ya había surgido en la Arqueología Evolutiva antes. Se encuentra detrás de la definición éxito replicativo de Leonard y Jones (1987) cuando hacen referencia al fitness darwiniano de los rasgos - tales como los artefactos - como diferente del éxito reproductivo de los individuos. La Arqueología Evolutiva también ha producido un número de estudios empíricos relacionados con el fitness de los rasgos - como artefactos - en los que es considerado totalmente por si mismo (i.e. Dunnell y Feathers 1991, O'Brien y Holland 1992, O'Brien et al. 1994).
Mientras que el concepto de éxito replicativo de Leonard y Jones nos permite acercar a la idea de que la selección actúa a un nivel infra-individual, los mismos Leonard y Jones (1987:216) rechazan esto, argumentando que el "replicative success of a particular trait might or might not affect the reproductive success of the bearer - those that do can be considered functional, and those traits with no selective import termed stylistic or neutral." En otras palabras, mientras los artefactos pueden exhibir diferentes valores en su fitness, ellos no pueden asumir el lógico status de individuos con capacidades reproductivas. Como otros arqueólogos evolutivos, Leonard y Jones aglutinan y conjugan a los artefactos con otros rasgos fisiológicos y conductuales como parte del fenotipo humano. La selección se mantiene en el nivel del individuo, así el éxito replicativo de un rasgo cultural y de otras características fenotípicas humanas son tan solo byproducts del éxito reproductivo diferencial de los individuos (Neff 1998).

Pero la visión del artefacto-como-fenotipo ignora el hecho de que, a diferencia de otras características fenotípicas, la reproducción de los artefactos y otros rasgos culturales no tienen nada que ver con el ciclo reproductivo de los individuos. Esta visión ha servido a la Arqueología Evolutiva adecuadamente, y no nos parece adecuado cambiarla. Sin embargo, Neff (1998) señala que la adopción acrítica de la visión del fenotipo extendido no ha permitido brindar una debida atención al hecho de que la herencia cultural y genética siguen diferentes canales, mientras ignoramos las mayores implicancias que para la evolución tienen estos dos distintos canales. Ya que la herencia cultural y la genética son completamente independientes una de otra, las genealogías dentro de las que se puede esperar observar descendencia con modificaciones son también completamente independientes una de la otra (Cullen 1993). Es más, las genealogías culturales no tienen relaciones especiales con los individuos, pero ya que múltiples genealogías culturales pueden estar pasando a través de un simple individuo, y la genealogía cultural relevante para un individuo en una fase de su vida puede ser completamente diferente de aquellas relevantes en otras fases de la misma.

Podemos poner estos dos puntos en una inferencia postulada por uno de nosotros (Neff 1998): ya que los artefactos exhiben valores diferentes en 
su éxito replicativo diferencial y que su reproducción es completamente autónoma de la reproducción de otras partes del fenotipo humano, la selección debe actuar al nivel del artefacto. Más específicamente, cualquier cosa que afecte la probabilidad de una transmisión exitosa de una instrucción cultural por sobre la manufactura y uso de algún artefacto o rasgo cultural es un agente de la selección, y puede causar un éxito replicativo diferencial de los rasgos que son transmitidos.

¿Cuáles son algunas de las implicancias de ver a la selección actuando debajo de nivel de individuo, y cuáles son las ventajas de adoptar esta perspectiva en la Arqueología Evolutiva? Primero, desde que las genealogías culturales no tienen relaciones especiales con los individuos, y que el interactor relevante es alguna entidad infraindividual, como el artefacto o un rasgo cultural dado, los individuos tienden a evaporarse en una insignificancia teórica y metodológica en la perspectiva aquí adoptada. Sin duda esto puede ser visto por alguno de nuestros colegas antropólogos - y arqueólogos también - como herejía en una ciencia que pretende estudiar al ser humano. Neff (1998) no lo ve de esta manera. ¿Qué más se puede pedir a una perspectiva teórica que nos dice que debemos focalizar nuestra atención en aquello que es observable y concreto en el registro arqueológico? Esto nos lleva mucho más allá de la suficiencia empírica. No necesitamos más "reconstruir" conductas desde los artefactos y luego aplicar nuestras teorías sobre las conductas reconstruidas; esta perspectiva nos dice que debemos analizar los artefactos o cualquier otro proceso o fenómeno arqueológico en estos términos cuando su diseño califica bien para persistir, por ejemplo, en términos de posibles variaciones en los valores del fitness. Por supuesto, esto es lo que venimos haciendo en la Arqueología Evolutiva de cualquier manera - i.e. O'Brien y Holland 1992 -, y que se ve en la propuesta de Lanata (1993a) y Lyman y O'Brien (1998) en aproximarnos al registro arqueológico a través de sus propiedades - tanto de las relacionales como las inherentes o inmanentes. Lo que sugerimos es que debemos explicitar las bases teóricas al focalizarnos en el diseño de los artefactos: esta base no es un marco evolutivo en el que la selección actúa en el nivel de los individuos o en el de un grupo de individuos sino que es más una teoría evolutiva en la que las diferencias del fitness están explícita- mente ligadas y/o sostenidas en residir en la escala de los rasgos culturales y que la selección afecta directamente la información contenida en el pool cultural.

\section{Transmisión y espacio}

Sin lugar a dudas podemos sostener que la Arqueología Evolutiva ha desarrollado tanto teórica como metodológicamente diferentes aspectos del cambio en las poblaciones humanas - aún cuando creemos que la discusión debe continuar. Quizás uno de los puntos más destacados está en centrar nuestras atención en el registro arqueológico y sus propiedades, reconociéndolo como nuestro objeto de estudio - ver supra. Esto ha permitido dejar de lado las explicaciones antropológicas del registro arqueológico que, como señala Dunnell (1989), son "timeless" y "spaceless." Sin duda, si el acercamiento es evolutivo, tiempo y espacio son dos variables que no pueden estar ausentes en nuestro análisis. Esta afirmación ha sido reforzada recientemente por O'Brien y Holland cuando destacan que el registro arqueológico "is a record of evolution" (1995b: 145) con información espacio-temporal específica, señalando los errores en que caen los "behavioral archaeologists" al no considerar estas propiedades del registro.

En un enfoque evolutivo, la concepción del tiempo como variable es un punto crucial en el estudio de la variabilidad del registro arqueológico. La naturaleza misma del tiempo arqueológico - i.e. un rango de $n$ años radiocarbónicos - nos inhibe de realizar cualquier inferencia de índole sistémica y/o sincrónica. Muy por el contrario, una de las peculiaridades con las que contamos los arqueólogos, es poder trabajar con rangos temporales y ver en ellos variabilidad y cambio en el registro arqueológico, es decir ver evolución. Y es de esta manera en donde el espacio comienza a tener un peso diferente en nuestras investigaciones. Sin embargo, debemos reconocer que no mucha atención hemos puesto los arqueólogos evolutivos en los aspectos espaciales del registro. Esto es válido aún cuando desde tiempo atrás, Dunnell y Dancey (1983) y Foley (1981a, b y c) comenzaron a valorar diferencialmente la propiedad espacial del registro arqueológico. Esta revalorización del espacio por parte de los arqueólogos evolutivos, nos per- 
mitirá ir más allá de las interpretaciones esencialistas, normativas y sistémicas de la explotación del espacio (Lanata 1993b). En este trabajo nos interesa remarcar el rol de espacio dentro de la transmisión cultural, en la perspectiva que aquí sostenemos.

Cuando anteriormente hablamos de la transmisión, señalamos que en la escala del artefacto, la selección de rasgos en su diseño resultan en fitness diferenciales y por lo tanto afectan el contenido del pool cultural. Esta de alguna manera es una de las escalas en la que opera la transmisión cultural. Otra escala que nos interesa discutir aquí - al menos para sentar las bases sobre las cuáles comprenderlas - se relaciona con las características del espacio como contenedor de una diversidad desconocida de genealogías-linajes.

La concepción del espacio en la Arqueología Evolutiva va más allá de las perspectivas sistémicas y conductuales que los estudios arqueológicos han mostrado tradicionalmente. Esto se debe a que el espacio es concebido unidimensionalmente - buscando determinados sectores de donde proviene algún recurso -, bidimensionalmente - estableciendo un patrón de asentamiento - o como una suma de patchs ambientales explotados - formulando un sistema de asentamiento y/o movilidad. Esto lleva a pensar que la variación espacio-temporal del registro arqueológico es distintiva y particular y que su generación no tiene relaciones con presiones - ya sean éstas ambientales, sociales, etc.. En estas visiones subyace por ejemplo que el uso y la utilización del espacio es esencialmente azarosa.

En este trabajo nos interesa destacar al espacio como una variable de estudio requerida per se en cualquier investigación evolutiva. El espacio es el entorno, el canal a través del cual se dan competencias y/o interacciones - tanto en el nivel intraespecífico como inter-específico - y que además contiene la información arqueológica de ellas. Ya que aquí estamos discutiendo distintas escalas de la transmisión cultural, tan solo expondremos aquellas relacionadas a nivel de nuestra especie.

La interacción y/o competencia entre distintas poblaciones se ven afectadas por las características del espacio en el cual tienen lugar, repercutiendo en la dispersión de los rasgos. Existen una diversidad de ambientes - desde aquellos muy heterogéneos a aquellos muy homogéneos - en los que el dinamismo de la transmisión se ve afectado por diferentes cirscunstancias. Estas características de los ambientes, conjuntamente con las del pool cultural, conjugan el uso y explotación de espacio de una manera particular.

En forma independiente de la característica particular del área en la cual reside circunstancialmente una población, está puede encontrarse limitada por espacios en los cuales no puede permanecer por diferentes circunstancias - i.e. falta de alimentos, condiciones ambientales adversas - que hacen que no puedan subsistir en ellas. Estas áreas son barreras (Cox y Moore 1985), aquellos espacios que deben ser cruzados, evitados y/o eludidos en su dispersión hacia otro no colonizado y/o no comprendidos dentro de su movilidad habitual. Básicamente, las barreras son espacios en los que la ocupación humana no es posible, siendo los ambientes que la rodean relativamente semejantes. Estas barreras limitan la dispersión de las poblaciones, produciendo un límite fijo en la misma, aunque no necesariamente tiene porque aislarlas.

En el uso y explotación del espacio es posible reconocer la existencia de diferentes pathways. Estos pathways son espacios en los cuales las poblaciones se mueven de un área a otra, de un sector a otro. El más común es el corredor (Cox y Moore 1985), y se caracteriza por incluir en él una serie de diferentes hábitats. Por sus características, el paso a través de los corredores no es difícil ya que por definición, conecta ambientes semejantes. A diferencia de esto, cuando los ambientes comunicados son distintos, esos pathways se convierten en filtros (Cox y Moore 1985, Burnery 1996, Lanata 1997). Un caso particular es aquel en el que el espacio de residencia de una población está rodeado por hábitats totalmente diferentes, y difíciles sortear. Por lo tanto las posibilidades que tiene una población de dispersarse y/o movilizarse a través de estos espacios son pocas y/o nulas, y están muy ligadas a la presencia de situaciones excepcionales que le permitan cruzarlos. Cuando esto se da estamos frente a sweeptakes y generalmente corresponde a un proceso originado en un simple suceso de colonización (Burney 1996).

Barreras, filtros, corredores y sweeptakes presentan situaciones diferentes en cuando a la posibilidad de transmitir diferencialmente rasgos de un pool cultural. Una barrera impide.el paso a través de ella de una población con un pool cultural $\alpha$. Esa 
población puede rodear la barrera, supongamos un desierto, lo que no produce ningún cambio de importancia. Tan solo esa barrera-el desierto-podrá ser superada con la aparición de nuevos rasgos, por lo que estarían ya en presencia de un pool cultural $\alpha$. En este caso, el nuevo rasgo puede estar ligado a i) la conservación de agua o ii) a tener la posibilidad de desplazarse a través de él. ${ }^{3} \mathrm{Al}$ suceder esto, surgen los corredores. En otras palabras para cruzar una barrera es necesario que tanto población e individuos diversifiquen levemente su pool cultural, generando nuevos rasgos que permitan franquearla. En el ejemplo mencionado, los pools culturales $\alpha$ y $\alpha^{\text {c no son }}$ sustancialmente diferentes ya que los ambientes que rodean el desierto son semejantes. Asi los pools culturales $\alpha$ y $\alpha^{\prime}$ presentan una diferencia temporal, que está solamente relacionada con la capacidad de poder utilizar un corredor determinado.

Cuando hablamos de filtros estamos ante una situación distinta. Mientras que los rasgos del pool cultural $\beta$ son útiles en uno de los ambientes de los extremos del filtro, no pasa lo mismo en el otro extremo. Si una población se dispersa de un ambiente a otro a través de un filtro, no todos los rasgos del pool cultural $\beta$ le serán útiles, por lo que además de generar nuevos rasgos se dejan de utilizar otros, dando lugar en el tiempo a un pool cultural $\beta$ '. En este caso, durante la dispersión de un ambiente a otro, la población va generando nuevos rasgos en su pool cultural a la vez que pierde otros, dando lugar al pool cultural $\beta^{\prime}$. Otro caso puede darse cuando dos poblaciones $\alpha$ y $\beta$ tienen contacto a través de un filtro. Aquí, rasgos de un pool cultural aparecen y/o aumentan sus frecuencias en el otro y viceversa. Es decir que el filtro contribuye a tamizar, sortear los rasgos de ambos pools que pasan de $\alpha$ a $\beta$, generando nuevos pools culturales $\alpha^{\prime \prime}$ у $\beta^{\text {". }}$.
Los sweeptakes representan fundamentalmente casos de aislamiento en donde por ejemplo los rasgos del pool cultural $\delta$ casi no han sufrido modificaciones a lo largo del tiempo. Es decir que se produjo la colonización de ese espacio y una barrera encierra a la población. El desarrollo de la población sigue pero sin sufrir inputs de otros pools culturales.

\section{Palabras finales}

Si admitimos que la selección y otros mecanismos evolutivos trabajan en un nivel infra-individual, en los que la variación cultural está implícita, podremos comenzar a ver las cosas de una manera diferente. Podremos buscar mejores métodos para identificar descendencias culturales comunes (i.e. Neff 1993 y 1998, Tschauner 1994), y por lo tanto poder monitorear los efectos de la selección a través del tiempo y del espacio en distintos linajes evolutivos culturales. Relaciones co-evolutivas entre diferentes tipos de artefactos o rasgos culturales podrán tener un rol explicativo más importante, y podremos comenzar a ver los conjuntos artefactuales arqueológicos como comunidades ecológicas, en las que determinadas tipos incluidos en las clases de artefactos constituyen condiciones ambientales relevantes hacia la evolución dirijida a otras clases de artefactos. Grupos de selección podrán ser re-definidos para referirse no a la selección actuando sobre grupos humanos, sino a la selección actuando en el fitness diferencial entre grupos o sub-conjuntos de artefactos. Al poner en juego la escala espacial de los análisis evolutivos vemos que se refuerza la idea de considerar la escala del artefacto como replicador o vehículo de la transmisión cultural. 
LANATA, J.L.: NEFF. H. Discutiendo algunas escalas de la transmisión cultural: artefacto y espacio. Rev. do Museu de Arqueologia e Etnologia, São Paulo, Suplemento 3: 47-56, 1999.

\section{Bibliografía}

BARTON, C.M.; CLARK, G.A. (Eds.)

1997 Rediscovering Darwin. Evolutionary Theory and Archaeological Explanation. Archaeological Papers of the American Anthropological Association Arlington, AAA, $\mathrm{N}^{\circ} 7$.

\section{BETTINGER, R.L.}

1991 Hunter-Gatherers. Archaeological and Evolutionary Theory. New York: Plenum Press.

BETTINGER, R.L.; EERKENS, J.

1997 Evolutionary implications of metrical variation in Great Basin projectile points. C.M. Barton; G.A. Clark (Eds.) Rediscovering Darwin. Evolutionary Theory and Archaeological Explanation. Archaeological Papers of the American Anthropological Association, Arlington, AAA, $\mathrm{N}^{\circ} 7$ : 177-191.

BOESCH, C.

1993 Aspects of transmission of tool-use in wild chimpanzee. K.R. Gibson; T. Ingold (Eds.) Tools, Language and Cognition in Human Evolution. Cambridge, Cambridge University Press: 171-183.

BONNER, J.T.

1980 The Evolution of Culture in Animals. Princeton: Princeton University Press.

BOONE, J.L.; SMITH, E.A.

1998 Is it Evolution yet? A critique of Evolutionary Archaeology. Current Anthropology, 39 (supplement): 141-173.

BOYD, R.; RICHERDSON, P.J.

1985 Culture and the Evolutionary Process. Chicago: University of Chicago Press.

BURNEY, D.A.

1996 Historical perspective on human-assited biological invasions. Evolutionary Anthropology, 4: 216-221.

COX, C.; MOORE, P.D.D.

1985 Biogeography: An Ecological and Evolutionary Approach. Oxford: Blackwell, 4th. edition.

CULLEN, B.

1993 The Darwinian resurgence and the cultural virus critique. Cambridge Archaeological Journal, 3 (2): 179-202.

DAWKINS, R.

1976 The Selfish Gene. Oxford: Oxford University Press.

1982 The Extended Phenotype. The Gene as the Unit of Selection. Oxford: W. H. Freeman and Company.

DUNNELL, R.C.

1978a Style and function: a fundamental dichotomy. American Antiquity, 43: 192-202.

1978b Natural selection, scale, and cultural evolution: some preliminary considerations. Trabajo presentado en el 77th Annual Meeting of the Society for American Archaeology, Los Angeles. Reprinted in M. J. O’Brien (Ed.) 1996.
Evolutionary Archaeology: Theory and Application. Salt Lake City, University of Utah Press: 24-29.

1980 Evolutionary theory and archaeology. Advances in Archaeological Method and Theory, 3: 35-99.

1989 Aspects of the Application of Evolutionary Theory in Archaeology. C.C. Lamberg-Karlovsky (Ed.) Archaeological Thought in America. Cambridge, Cambridge University Press: $35-49$

1992a Archaeology and evolutionary science. LuAnn Wandsnider (Ed.) Quandaries and Quests: Visions of Archaeology's Future. Center for Archaeological Investigations, Carbondale, Occasional Paper $\mathrm{N}^{\circ}$ 20: 209-224.

1992 b Is a scientific archaeology possible? L. Embree (Ed.) Metaarchaeology. Netherlands, Kluwer Academic Publisher: 75-97.

1994 Why is there a hunter-gatherer archaeology? J.L. Lanata; L.A. Borrero (Eds.) Arqueologia de Cazadores-Recolectores. Límites, Casos y Aperturas. Arqueología Contemporánea, Buenos Aires, 5: 7-15.

DUNNELL, R.C.; FEATHERS, J.K

1991 Late Woodland manifestations of the Malden Plain, southeast Missouri. M.S. Nassaney; C.R. Cobb (Eds.) Stability, Transformation, and Variation: The Late Woodland Southeast. New York, Plenum Press: 21-45.

DUNNELL, R.C.; DANCEY, W.S.

1983 The siteless survey: a regional scale data collection strategy. Advances in Archaeological Method and Theory, 6: 267-287.

FOLEY, R.

1981a Off-site archaeology: an alternative approach for the short-sited. I. Hodder; G. Isaac; N. Hammond (Eds.) Pattern of the Past: Studies in Honour of David Clarke. Cambridge, Cambridge University Press: 157-183.

1981b A model of regional archaeological structure. Proceedings of the Prehistoric Society, 47: 1-17.

1981c Off-site Archaeology and Human Adaptation in Eastern Africa. An analysis of regional artefact density in the Amboseli, Southern Kenya. Cambridge Monographs in African Archaeology 3. BAR International Series 97, Oxford.

1989 The ecology of speciation: Comparative perspectives on the origins of modern humans. P.A. Mellars; C.B. Stringer (Eds.) The Human Revolution. Behavioral and Biological Perspectives on the Origins of Moderns Humans. Edinburgh, Edinburgh University Press: 298320.

GRIFFIN, D.R.

1984 Animal Thinking. Cambridge: Harvard Univesity Press. 
LANATA, J.L.; NEFF, H. Discutiendo algunas escalas de la transmisión cultural: artefacto y espacio. Rev. do Museu de Arqueologia e Etnologia, São Paulo, Suplemento 3: 47-56, 1999.

HULL, D.L.

1980 Individuality and Selection. Annual Review of Ecology and Systematics, 11: 311-332.

JONES, G.T.; LEONARD, R.D.; ABBOTT, A.L.

1995 The structure of selectionist explanations in archaeology. P. Teltser (Ed.) Evolutionary Archaeology. Methodological Issues. Tucson, University of Arizona Press: 13-32.

KIRCH, P.

1980 The archaeological study of adaptation: theoretical and methodological issues. Advances in Archaeological Method and Theory, 3: 101-148.

KROEBER, A.L.

1931 Historical reconstruction of culture growths and organic evolution. American Anthropologist, 33: 149-157.

LANATA, J.L.

1993a The evolution of human adaptation in Southern South America. Current Perspectives. Trabajo presentado en el 58th Annual Meeting of the Society for American Archaeology. St. Louis.

1993b Evolución, espacio y adaptación en grupos cazadores-recolectores. Revista do Museu de Arqueologia e Etnologia, São Paulo, 3: 3-15.

1997 Algunos Desafios de la Arqueologia Evolutiva. Trabajo presentado en el IX Congreso Nacional de Arqueología Uruguaya, Colonia.

1998 Las diferencias en la Arqueología Evolutiva hoy. Trabajo presentado en la 1a. Reunião Internacional de Teoria Arqueológica na América do Sul. Vitória.

LEONARD, R.D.; JONES, G.T

1987 Elements of an Inclusive Evolutionary Model for Archaeology. Journal of Anthropological Archaeology, 6: 199-219.

LYMAN, R.L.; O'BRIEN, M.J.

1998 The goals of Evolutionary Archaeology: History and explanation. Current Anthropology, 39 (in press).

LYMAN, R.L.; O'BRIEN, M.J.; DUNNELL, R.C.

1997 The Rise and Fall of Culture History. New York: Plenum Press.

MASCHNER, H.D.G. (Ed.)

1996 Darwinian Archaeologies. NY: Plenum Press.

Mc GREW, W.C.

1992 Chimpanzee Material Culture. Implications for Human Evolution. Cambridge: Cambridge University Press.

1993 The Intelligent Use of Tools: Twenty propositions. K.R. Gibson; T. Ingold (Eds.) Tools, Language and Cognition in Human Evolution. Cambridge, Camdridge University Press: 151-170.

NEFF, $\mathrm{H}$.

1992 Chemical Characterization of Ceramic Pastes in Archaeology. Madison: Prehistory Press.

1993 Theory, Sampling, and Technical Analysis in archaeological pottery analysis. American Antiquity, 58: 23-44.
1998 Differential Persistence of What? The Scale of Selection Issue in Evolutionary Archaeology. Trabajo presentado en el 63th Annual Meeting of the Society for American Archaeology, Seattle.

NEFF, H.; ARROYO, B.

s.d. The evolution of Early Formative ceramic traditions in Pacific coastal southern Mesoamerica. Proceedings of the 1996 Chacmool Conference (en prensa).

NEFF, H.; LARSON, D.O.

1997 Methodology of comparison in Evolutionary Archaeology. C.M. Barton; G.A. Clark (Eds.) Rediscovering Darwin. Evolutionary Theory and Archaeological Explanation. Archaeological Papers of the American Anthropological Association, Arlington, AAA, $\mathrm{N}^{\circ} 7: 75$ 94.

O'BRIEN, M.J.

1996 Evolutionary Archaeology. Theory and Application. Salt Lake: University of Utah Press.

O'BRIEN, M.J.; HOLLAND, T.D.

1990 Variation, selection, and the archaeological record. Archaeological Method and Theory, 2: 31-79.

1992 The role of adaptation in archaeological explanation. American Antiquity, 57: 36-59.

1995a The nature and premise of a selection-based archaeology. P. Teltser (Ed.) Evolutionary Archaeology. Methodological Issues. Tucson, University of Arizona Press: 175-200.

1995b Behavioral archaeology and the extended phenotype. J.M. Skibo; W.H. Walker; A. Nielsen (Eds.) Expanding Archaeology. Salt Lake, University of Utah: 143-161.

O'BRIEN, M.J.; HOLLAND, T.D.; HOARD, R.J.; FOX, G.L.

1994 Evolutionary implications of design and performance characteristics of prehistoric pottery. Journal of Archaeological Method and Theory, 1: 259-304.

RINDOS, D.

1984 The Origins of Agriculture: An Evolutionary Perspective. New York: Academic Press.

1985 Darwinian selection, symbolic variation and the evolution of culture. Current Anthropology, 26: 65-88.

1989a Diversity, variation and selection. R.D. Leonard; G.T. Jones (Eds.) Quantifying Diversity in Archaeology. Cambridge, Cambridge University Press: 13-24.

1989 b Undirected variation and the Darwinian Explanation of Cultural Change. Archaeological Method and Theory, 1:1-45.

SCHIFFER, M.B.

1996 Some relationships between behavioral and evolutionary archaeology. American Antiquity, 61: 643-662.

SHOTT, M.J.

1997 Transmission theory in the study of stone tools. A Midwestern North America exam- 
LANATA, J.L.; NEFF, H. Discutiendo algunas escalas de la transmisión cultural: artefacto y espacio. Rev. do Museu de Arqueologia e Etnologia, São Paulo, Suplemento 3: 47-56, 1999.

ple. C.M. Barton; G.A. Clark (Eds.) Rediscovering Darwin. Evolutionary Theory and Archaeological Explanation. Archaeological Papers of the American Anthropological SMITH, E.A. Association, Arlington, AAA, N² 193-204.

1991 Inujjuamiut Foraging Strategies. Evolutionary Ecology of an Arctic Hunting Economy. New York: Aldine de Gruyter.

SMITH, E.A.; WINTERHALDER, B.

1992 Evolutionary Ecology and Human Behavior. New York: Aldine de Gruyter.

SPENCER, C.S

1997 Evolutionary approach in archaeology. JourTELTSER, P. (Ed.)
1994 Archaeological Systematics and Cultural Evolution: Retrieving the Honour of Culture History. Man (n.s.) 29: 77-93.

1995 Evolutionary Archaeology. Methodological Issues. Tucson, Tschauner, Hartmut: University of Arizona Press.

\section{VISALBERGHI, E.}

1993 Capuchin Monkeys: A Window into Tool Use in Apes and Humans. K.R. Gibson; T. Ingold (Eds.) Tools, Language and Cognition in Human Evolution. Camdridge, Cambridge University Press: 138-150.

WALKER, S.

1983 Animal Thought. London: Routledge and Kegan Paul. 